\title{
Downregulation of Ascl2 promotes cell apoptosis by enhancing autophagy in colorectal cancer cells
}

\author{
Huipeng Wang", Tao Ye", Yuankun Cai, Wenjie Chen, Hongwei Xie*, Chongwei Ke* \\ Department of General Surgery, The Fifth People's Hospital of Shanghai, Fudan University, Shanghai, China \\ Contributions: (I) Conception and design: H Wang, C Ke; (II) Administrative support: T Ye; (III) Provision of study materials or patients: W Chen; \\ (IV) Collection and assembly of data: H Xie; (V) Data analysis and interpretation: H Wang, C Ke; (VI) Manuscript writing: All authors; (VII) Final \\ approval of manuscript: All authors. \\ \#These authors contributed equally to this work as co-first authors. \\ *These authors contributed equally to this work as co-correspondence authors. \\ Correspondence to: Dr. Chongwei Ke; Dr. Hongwei Xie. Department of General Surgery, The Fifth People's Hospital of Shanghai, Fudan University, \\ 801 Heqing Road, Shanghai 200240, China. Email: dr_kecw@163.com; 13816819761@163.com.
}

\begin{abstract}
Background: Colorectal cancer (CRC) is the third most common cancer, according to recently published literature. While the incidence and the mortality of CRC has decreased due to effective cancer screening measures, there has been an increase in the number of young patients diagnosed with colon cancer due to unclear reasons. As a target molecule of the Wnt signaling pathway, Ascl2 is an important marker of CRC stem cells and plays an important role in maintaining the nature of colon cancer stem/precursor cells. However, the role of Ascl2 in autophagy in CRC cells is rarely elucidated.

Methods: In this study, we found that Ascl2 was increased in CRC compared with adjacent tissue. Downregulation of Ascl2 in CRC cells could suppress proliferation and invasion, and induce apoptosis, of CRC cells. Moreover, we found that autophagy-relative protein LC3 increased after Ascl2 knockdown. Furthermore, we treated CRC cells with autophagy inhibitors 3-MA (3-Methyladenine) and CQ (Chloroquine).

Results: The results showed that autophagy inhibitors could prevent apoptosis, which was induced by Ascl2 knockdown. Finally, we confirmed that the downregulation of Ascl2 in CRC cells could alleviate the pathological process in vivo by xenograft experiment.

Conclusions: Our findings indicated that si-Ascl2 (small/short interfering) exerted a tumor suppression function in CRC by inducing autophagic cell death, and suggest that Ascl 2 targeted therapy represents a novel strategy for CRC treatment.
\end{abstract}

Keywords: Autophagy; Ascl2; apoptosis; colorectal cancer (CRC)

Submitted Mar 10, 2021. Accepted for publication Apr 20, 2021.

doi: 10.21037/jgo-21-183

View this article at: http://dx.doi.org/10.21037/jgo-21-183

\section{Introduction}

In 2020, approximately 935,000 people died from colorectal cancer (CRC) and more than 1.9 million new CRC patients were diagnosed. Overall, CRC ranks third in terms of incidence of malignant tumors in the world, but second in terms of mortality (1). However, progress in the treatment of CRC has been limited. New drugs have roughly doubled the average survival time for advanced CRC over the past decade, but patients usually die within 3 years of being diagnosed (2). Accumulating evidence indicates that various genetic and epigenetic changes could activate multiple oncogenic critical signaling pathways for the pathogenesis of CRC, such as $W n t / \beta$-catenin signaling pathway (3). The activation of this pathway could initiate a transcriptiondependent oncogenic process to promote cell proliferation 
and apoptotic resistance.

Ascl2 is a key downstream molecule of the Wnt/ $\beta$-catenin signaling pathway. It is a basic helix-loop-helix transcription factor homolog (4). Ascl2 is mainly found in the intestine cells where it plays an indispensable role in the maintenance of intestinal stem cells (5). It has been reported that Ascl2 acts as a phenylbutyrate resistance-related gene in CRC (6). However, this has been rarely reported in the literature. The role of Ascl2 which keep the self-renewal of CRC progenitor cells may through up-regulation of STAT3 expression level and synergistic interaction with LIF phosphorylation STAT3 signaling pathway, as well as entering into the regulatory network of embryonic stem cells through TCF3. Shang et al. confirmed that HIF-1 $\alpha$ (hypoxia inducible factor-1) could induce the expression of Ascl2 to inhibit the production of miR-200b to promote epithelial-mesenchymal transition in CRC (7). It was reported that, Ascl2 was a downstream target of HIF-1 $\alpha$ and had a critical role in the EMT phenotype induced by hypoxia or HIF-1 $\alpha$ over-expression. However, the effects of Ascl2 in the autophagy of CRC remain elusive.

Autophagy refers to the process of inducing intracellular substances to be produced and transported to lysosomal degradation through a series of pathways under the influence of external adverse environmental factors such as starvation, oxidative stress, infection and nutritional deficiency (8). A number of studies have linked autophagy to various cancers, and it could either enhance or prevent tumorigenesis $(9,10)$. Autophagy is excessively activated in the intestinal epithelium of patients and mouse models of CRC (11). Autophagosomes are actively produced and promptly consumed in human CRC cell lines (12). Autophagy is essential for maintaining cellular homeostasis and supporting cell survival and growth (13). However, the underlying molecular mechanisms of autophagy in CRC remain elusive.

There are few relevant studies on the relationship between ASCL2 and autophagy, the effect on the occurrence and development of colorectal cancer, and the mechanism. We use the correlation between them to solve one of the key links in the pathogenesis of colorectal cancer, which may provide more effective guiding value for the clinical treatment of colorectal cancer. In the present study, we further confirmed that Ascl2 expression is elevated in CRC. Therefore, we downregulated Ascl2 in CRC cells and found that the proliferation and invasion of CRC cells were attenuated, but apoptosis progressed. To explore the role of Ascl2 in the autophagy of CRC, we detected the expression of LC3 and p62 after Ascl2 knockdown.

We present the following article in accordance with the ARRIVE reporting checklist (available at http://dx.doi. org/10.21037/jgo-21-183).

\section{Methods}

All procedures performed in this study involving human participants were in accordance with the Declaration of Helsinki (as revised in 2013). The study was approved by Institutional Ethics Committee of The Fifth People's Hospital of Shanghai, Fudan University (No.: 2017-097) and informed consent was taken from all the patients. Animal experiments were performed under a project license (No.: 20171254A184) granted by Department of Laboratory Animal Science, Fudan University, in compliance with Fudan University guidelines for the care and use of animals.

In this study, we will continue to verify the expression of ASCL2 in colorectal cancer specimens, conduct cell and in vivo animal experiments, and study the relationship between ASCL2 and colorectal cancer, as well as the correlation and mechanism between ASCL2 and autophagy.

\section{Clinical specimens}

Tissue specimens from 7 CRC patients were collected at The Fifth People's Hospital of Shanghai, Fudan University. The patients were pathologically diagnosed with CRC and provided informed consent to participate in the study.

\section{Cell culture and transfection}

HT-29 cells were purchased from American Type Culture Collection (Manassas, VA, USA). HT-29 cells were cultured in Dulbecco's modified Eagle's medium (Hyclone, Logan, UT, USA) with $10 \%$ heat-inactivated fetal bovine serum (Gibco, USA). HT-29 cells were placed in plates with humidified air by $5 \% \mathrm{CO}_{2}$ at $37^{\circ} \mathrm{C}$.

The si-control and si-Ascl2, which were purchased from RiboBio (Guangzhou, China), were transfected into HT-29 cells by Lipofectamine 3000 (Thermo Fisher Scientific, USA), according to the manufacturer's instructions. Briefly, when the cell density was about $80 \%$, Lipofectamine 2000 and silencer siRNAs were solvated by opti-MEM (Thermo Fisher Scientific, USA), respectively, and silencer siRNA mixture was slowly added to the Lipofectamine 2000 mixture. All the mixtures were then added to the cells after resting for $15-$ 
Table 1 Primers for quantitative reverse transcription polymerase chain reaction

\begin{tabular}{lll}
\hline Gene name & Polarity & Sequence \\
\hline Ascl2 & Forward & GCGTGAAGCTGGTGAACTTG \\
& Reverse & GGATGTACTCCACGGCTGAG \\
GAPDH & Forward & TCCACCCATGGCAAATTCCA \\
& Reverse & GCCTTCTCCATGGTGGTGAA \\
\hline
\end{tabular}

$20 \mathrm{~min}$. After $48 \mathrm{~h}$, we continued with the experiments.

\section{Western blotting}

Cells were harvested by RIPA lysis buffer (Biolegend, USA), according to manufacturer's instructions. The protein concentration was detected by bicinchoninic acid kits (Thermo Fisher Scientific, USA). Proteins were separated by adding to sodium dodecyl sulfate-polyacrylamide gel electrophoresis. Then proteins were transferred to polyvinylidene difluoride membranes (GeneTex, USA). After washing with phosphate-buffered saline (PBS) for $5 \mathrm{~min}$, the membranes were blocked by $5 \%$ skim milk for $1 \mathrm{~h}$ at room temperature. Then the membranes were incubated in primary antibodies at $4{ }^{\circ} \mathrm{C}$ overnight. After washing with phosphate-buffered saline containing $0.05 \%$ Tween-20 for $30 \mathrm{~min}$, the membranes were incubated in secondary antibodies for $1 \mathrm{~h}$. Finally, the signals were observed using chemiluminescent enhanced chemiluminescence reagent (Thermo Scientific, USA) and Ascl2 antibody (Abcam, UK).

\section{Quantitative reverse transcription polymerase chain reaction ( $q R T-P C R)$}

Cells were harvested by RNAiso Plus (TaKaRa, JPN), according to the manufacturer's instructions. The PrimeScript RT Reagent Kit (TaKaRa, JPN) was then used to obtain cDNA as qRT-PCR templates; qRT-PCR was performed by SYBR Premix Ex Taq (TaKaRa, JPN), and GAPDH served as the internal control. All the experiments were carried out in triplicate. The sequences of the primers are listed in Table 1.

\section{Cell proliferation assays}

Cells were seeded in a 96-well plate. When the cell density was approximately $80 \%$, cells were transfected with si- control or si-Ascl2. Cell proliferation was detected by Cell Counting Kit-8 (CCK-8; Dojindo, USA), according to the manufacturer's instructions.

\section{Colony formation assay}

Cells were transfected with si-control or si-Ascl 2 and seeded in a 6-well plate for 1,000 cells per well. After 2 weeks, the cells were fixed by $4 \%$ paraformaldehyde for $15 \mathrm{~min}$. After washing with PBS for $20 \mathrm{~min}$, cells were stained by crystal violet for 10-15 min. Pictures of the cells were then taken.

\section{Scratch assay}

Cells were transfected with si-control or si-Ascl2 and seeded in a 6-well plate. When the cell density approached 90\%, the cells were scratched vertically with a P1000 pipette tip. Wells were then washed with PBS to remove cellular debris. The same fields were photographed at 0 and $48 \mathrm{~h}$. The area of the wound was calculated with Image-Pro Plus 6.0 (Media Cybernetics, USA).

\section{Apoptosis assay}

Cell apoptosis was detected by Annexin V-fluorescein isothiocyanate (FITC)/propidium iodide (PI) kit (BD, USA). Briefly, cells were digested by pancreatic ferment and collected in PBS. Cells were washed with PBS and resuspended in $100 \mu \mathrm{L} 1 \times$ buffer. In total, $5 \mu \mathrm{L}$ Annexin $\mathrm{V}$-FITC and PI were added. After $15 \mathrm{~min}, 400 \mu \mathrm{L} 1 \times$ buffer was added to the mixture to detect apoptotic cells by flow cytometry.

\section{Immunobistochemical assay}

Tumors were retrieved from animals and washed, dehydrated, and embedded in paraffin. Tumors were cut into $4-\mu \mathrm{m}$-thick slices. The slices were then deparaffinized and treated with antigen retrieval solution. The sections were stained with cleaved caspase 3 or Ki-67 antibody (CST, USA). The expression of cleaved caspase 3 and Ki-67 in tissues was observed with a microscope (Leica Biosystems, Germany).

\section{In vivo model of CRC}

Cells were infected with sh-control or sh-Ascl2 virus to construct a stable cell line. Male nude mice (4-5 weeks old) 

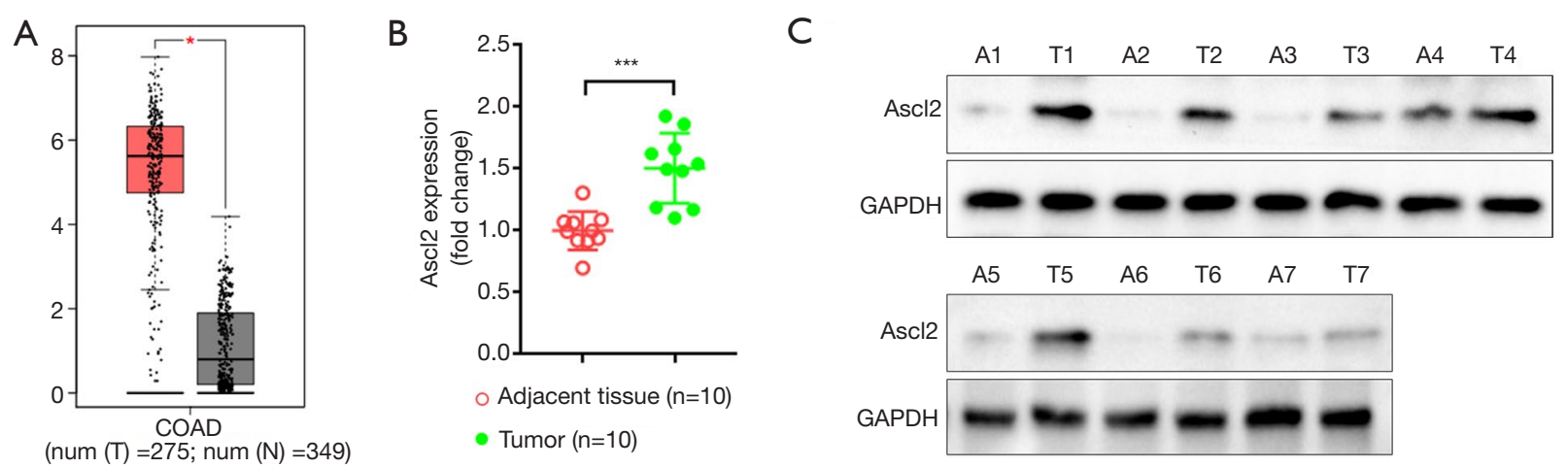

Figure 1 Expression of Ascl2 is elevated in colorectal cancer (CRC). (A) Analysis of the expression of Ascl2 in CRC using the Gene Expression Profiling Interactive Analysis website; (B) quantitative reverse transcription polymerase chain reaction assay was used to detect Ascl2 expression of in colorectal tumors and adjacent tissues; (C) Western blot assay was used to detect Ascl2 expression in colorectal tumors and adjacent tissues. All data are presented as mean \pm standard deviations from 3 independent experiments. ${ }^{* * *}, \mathrm{P}<0.001$. A, adjacent tissue; $\mathrm{T}$, tumor.

were injected with $5 \times 10^{6}$ of the stable cells subcutaneously. After 5 days, the volume of the tumors was measured every 3 days. At 20 days, tumors were collected from the animals and weighed, and then stored in $4 \%$ formalin. Tumor volume was calculated as $\left(\right.$ length $\times$ width $\left.^{2}\right) / 2$.

\section{Statistical analysis}

All data were analyzed with SPSS 21.0 software (SPSS, Armonk, NY, USA). Quantitative variable differences between groups were analyzed $t$-test. Fisher's exact test and $\chi^{2}$-test were used for categorical variables. Kaplan-Meier was used to analysis of CRC patients survival curves. $\mathrm{P}<0.05$ indicated statistical significance.

\section{Results}

\section{Elevated expression of Ascl2 in CRC}

It has been reported that Ascl2 is upregulated in intestinal neoplasia (14). We further explored the expression of Ascl2 in CRC. First, we analyzed the CRC data in The Cancer Genome Atlas by using the Gene Expression Profiling Interactive Analysis website and found that Ascl2 was significantly upregulated in CRC compared with normal tissues (Figure 1A). We then detected the expression of Ascl2 in patients with CRC. The qRT-PCR results showed that the mRNA levels of Ascl2 in CRC were elevated (Figure 1B). Finally, Western blot assay was performed to detect the protein expression of Ascl2. As shown in
Figure 1C, Ascl2 expression significantly increased in CRC.

\section{Downregulation of Ascl2 suppresses proliferation and migration, and induces apoptosis, in CRC cells}

To determine the effect of Ascl2 on the proliferation of CRC cells, we transfected HT-29 cells with si-control or siAscl2. qRT-PCR and Western blot assay were performed to monitor the knockdown effects. The results showed that the mRNA and protein levels of Ascl2 expression both decreased (Figure 2A). We subsequently evaluated the effect of si-Ascl2 on the proliferation of HT-29 cells by CCK8 assay. As shown in Figure 2B, Ascl2 downregulation could alleviate the proliferation of HT-29. We then used colony formation assay to further confirm the function of Ascl2 on proliferation. As shown in Figure $2 C$ and $2 D$, the colony numbers were fewer in the Ascl2 knock down group. To determine if Ascl2 knockdown would suppress the migration of HT-29, we used scratch tests. The results showed that Ascl2 downregulation could significantly inhibit the migration of HT-29 (Figure 2E,F). Finally, to verify whether Ascl2 could influence HT-29 apoptosis, we used flow cytometry to detect apoptotic cells. As shown in Figure $2 G$ and $2 H$, the reduced expression of Ascl2 led to increased apoptosis in HT-29 cells. The si-Ascl2-induced apoptotic events were associated with activation of cleaved caspase 3 (Figure 2I).

These results suggest that si-Ascl2 could inhibit proliferation and migration, and induce apoptosis, in HT29 cells. 
A

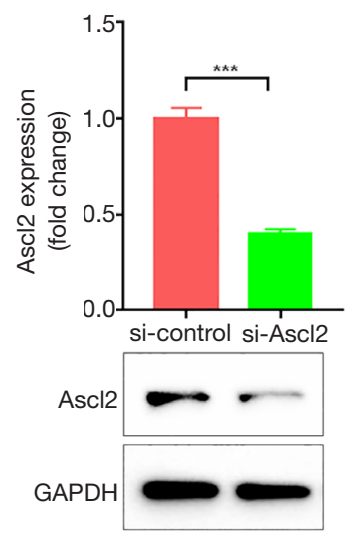

D

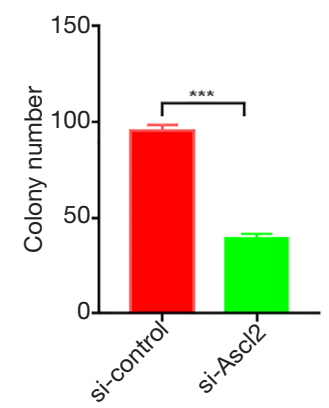

B

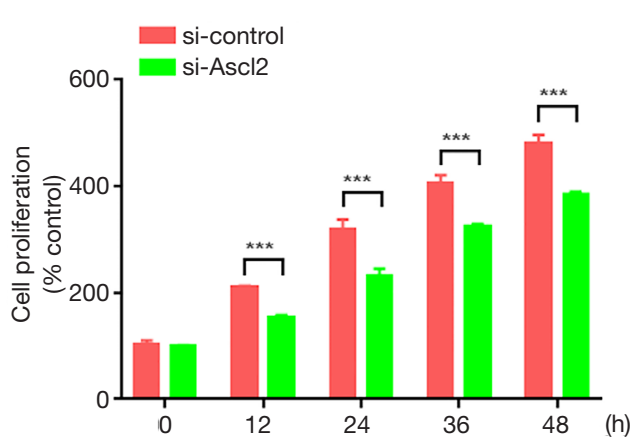

E

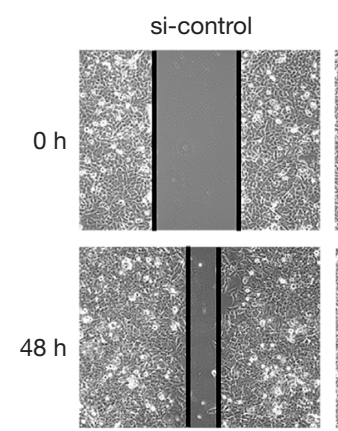

$\mathrm{H}$
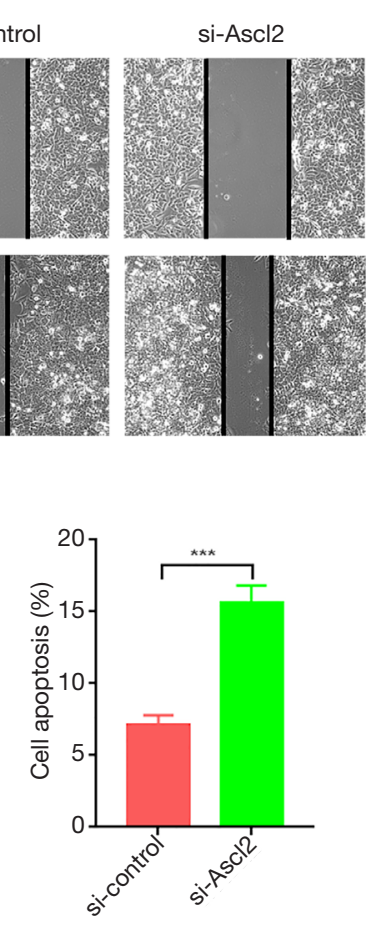

C

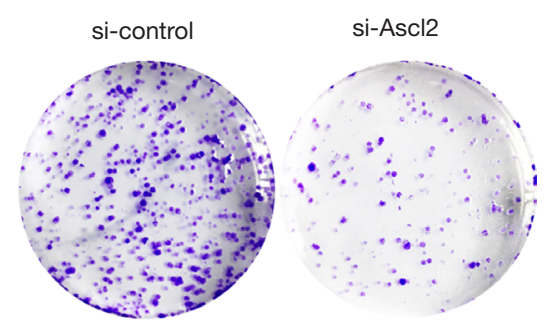

F

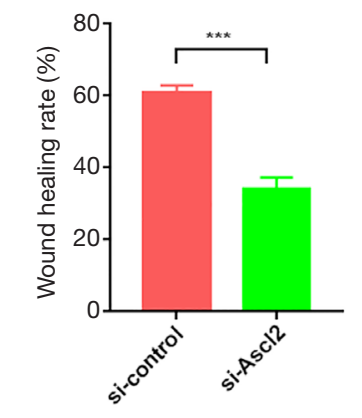

I

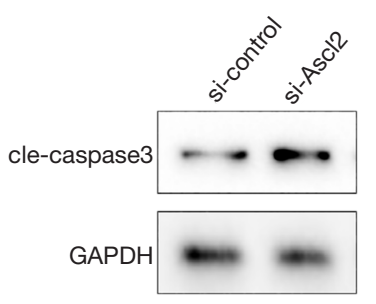

F

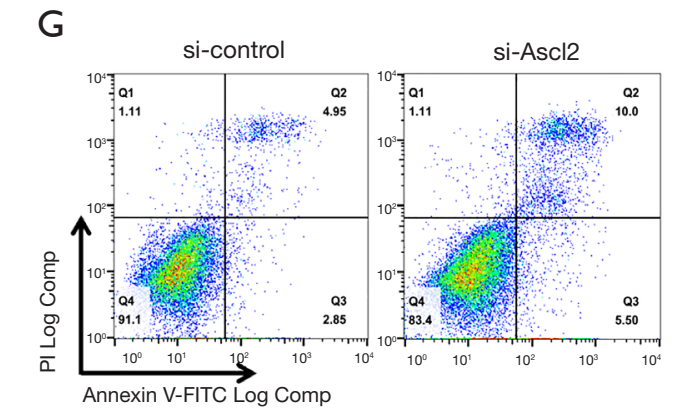

Figure 2 Downregulation of Ascl2 inhibited HT-29 proliferation and promoted apoptosis. (A) To confirm the knockdown efficiency of si-Ascl2 by quantitative reverse transcription polymerase chain reaction and Western blot assays; (B) HT-29 cells were transfected with si-control or si-Ascl2 to detect proliferation using Cell Counting Kit-8; (C,D) colony formation assay was performed to detect cell proliferation (crystal violet staining, 10×10); (E,F) scratch test was performed to detect cell migration $(10 \times 10) ;(\mathrm{G}, \mathrm{H})$ after transfection with si-control or si-Ascl2 for 48 h, HT-29 cell apoptosis was determined by ANXA5 (Annexin A5) and propidium iodide staining; (I) Western blot was performed to detect the expression of cleaved caspase 3 . All data are presented as means \pm standard deviations from 3 independent experiments. ${ }^{* * *}, \mathrm{P}<0.001$.

\section{Downregulation of Ascl2 induces apoptosis by promoting autophagy in HT-29 cells}

Previously published studies have suggested that autophagy plays essential roles in the pathological process of CRC (15-17). To confirm whether Ascl2 could mediate autophagy in CRC, we detected the expression of autophagy proteins, such as LC3 and p62. As shown in Figure 3A, LC3 was upregulated and p62 was downregulated when HT-29 cells were transfected with si-Ascl2. Furthermore, HT-29 cells were treated with autophagy inhibitors 3-methyladenine (3-MA) and chloroquine (CQ) to examine the effect of 
A

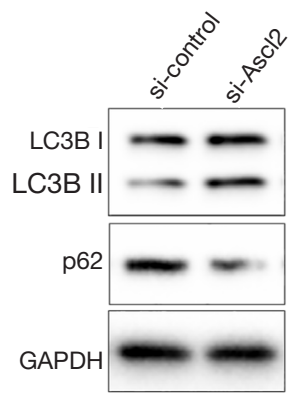

D

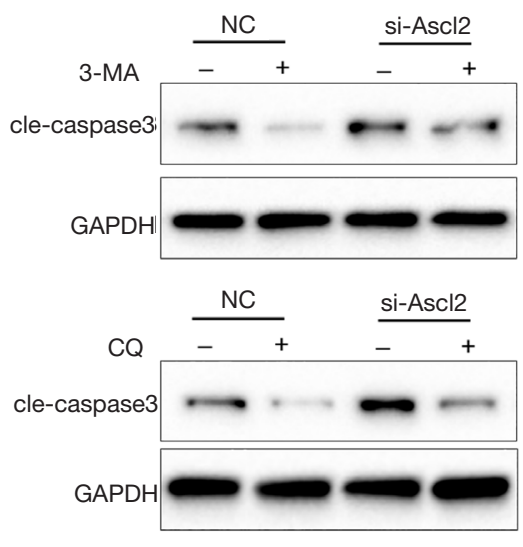

B

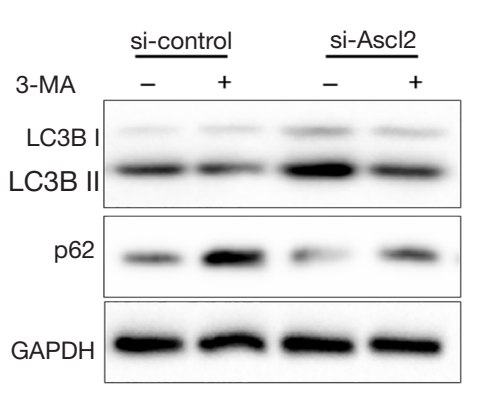

E
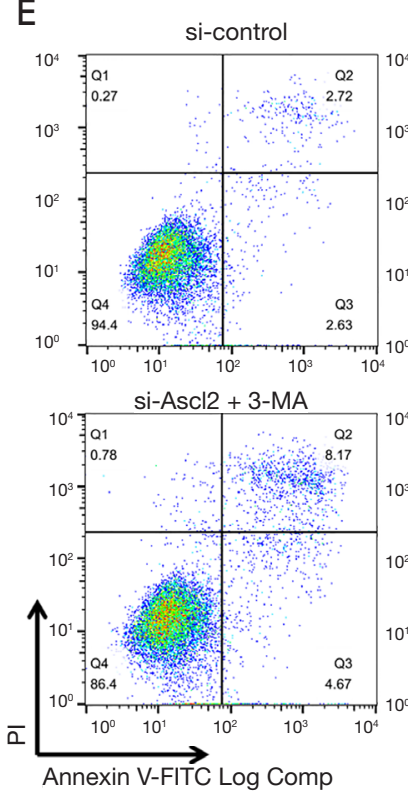

C

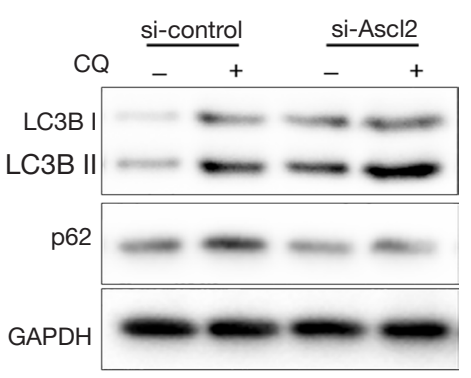

$\mathrm{F}$

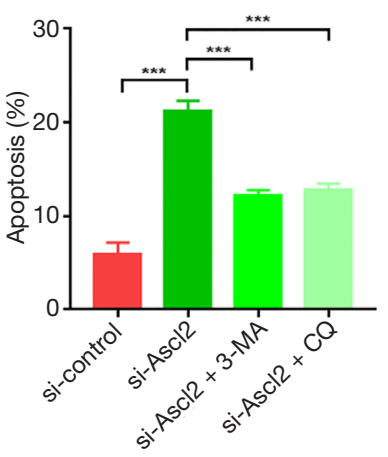

Figure 3 Downregulation of Ascl2 induced apoptosis by promoting autophagy in HT-29 cells. HT-29 cells were transfected with si-control or si-Ascl2 for 48 h (A) Analysis of the expression of LC3 and p62 by Western blot; (B) HT-29 cells were treated with 3-methyladenine (3-MA) to detect LC3 and p62 by Western blot; (C) HT-29 cells were treated with chloroquine (CQ) to detect LC3 and p62 by Western blot; (D) HT-29 cells were treated with 3-MA or CQ to detect cleaved capase 3 by Western blot; (E,F) ANXA5 (Annexin A5) and propidium iodide staining was performed to detect apoptotic cells after treatment with 3-MA or CQ in HT-29 cells. All data are presented as means \pm standard deviations from 3 independent experiments. ${ }^{* * *}, \mathrm{P}<0.001$.

Ascl2 on autophagy. As shown in Figure $3 B$ and $C$, treatment with either 3-MA or CQ significantly blocked autophagy in si-Ascl2-treated cells. Moreover, as shown in Figure 3D,E,F, the cell death induced by Ascl2 downregulation could be inhibited by autophagy inhibitors, which was supported by decreased cleaved caspase 3 expression and a significant decrease in the ANXA5 (Annexin A5)-positive cell fraction in HT-29 cells.

These findings indicate that autophagy is the major mechanism in the process of si-Ascl2-induced cell death in HT-29 cells.

\section{Downregulation of Ascl2 suppresses the proliferation of CRC in vivo}

Finally, to assess the role of Ascl2 in vivo, we constructed Ascl2 downregulation stable cell lines using sh-Ascl2. Stable cell lines were injected subcutaneously into nude mice to establish a xenograft model. As shown in Figure $4 A$ and $B$, compared with the si-control groups, si-Ascl2 led to slow proliferation of HT-29 cells in vivo. We also evaluated the effect of sh-Ascl2 in vivo by detecting the expression of cleaved caspase 3 and $\mathrm{Ki}-67$ in human CRC xenografts harvested from treated mice. The results showed that Ascl2 
A

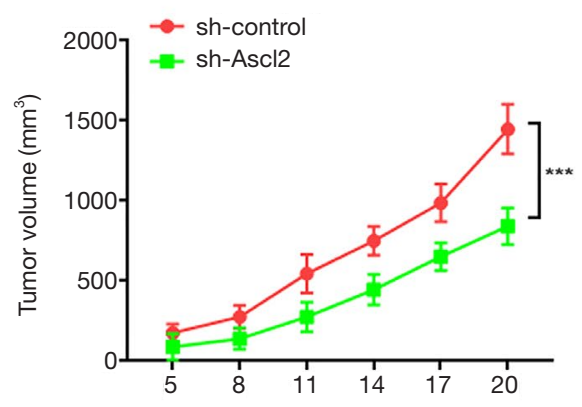

C

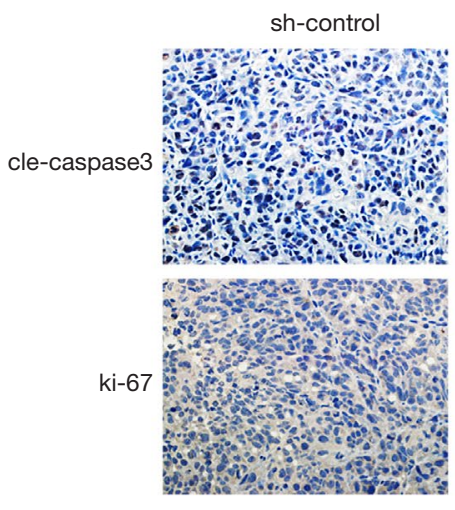

B

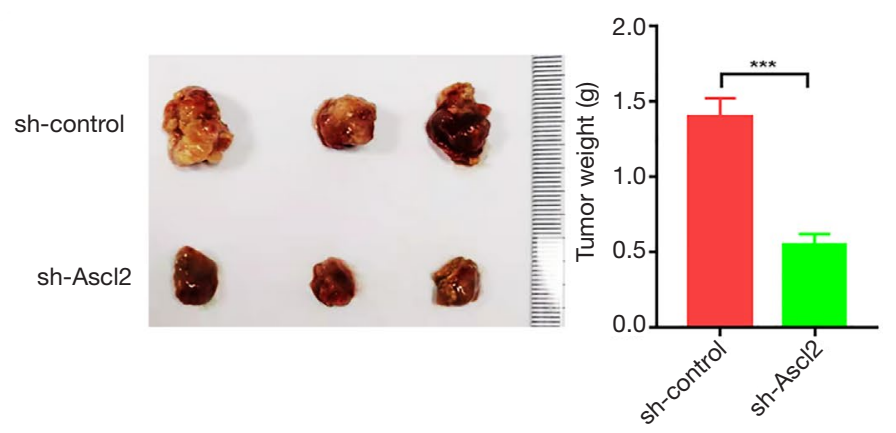

D D

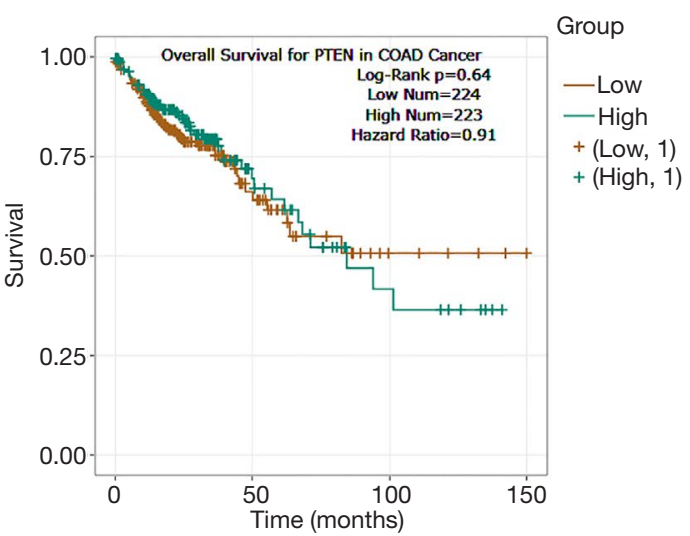

Figure 4 Downregulation of Ascl2 suppressed the proliferation of colorectal cancer (CRC) in vivo. (A) Growth curves of tumors derived from sh-control or sh-Ascl2 injected subcutaneously into nude mice; (B) tumors harvested from nude mice were weighed; (C) immunohistochemical assay was performed to detect cleaved caspase 3 and Ki-67 in tumors (40×10); (D) Kaplan-Meier analysis of Ascl2 expression in CRC based on The Cancer Genome Atlas. All data are presented as means \pm standard deviations from 3 independent experiments. ${ }^{* * *}, \mathrm{P}<0.001$.

downregulation significantly increased the number of cleaved caspase 3-positive CRC cells and decreased Ki-67positive CRC cells compared with the controls (Figure $4 C$ ).

Previous work has confirmed that Ascl2 could accelerate CRC. Furthermore, we found that the high expression of Ascl2 in CRC patients was associated with shorter progression-free survival by Kaplan-Meier survival analysis (Figure 4D).

\section{Discussion}

Colorectal malignancies are one of the common malignant tumors in the digestive system. Due to the development and clinical application of chemotherapeutic drugs and targeted drugs $(18,19)$, disease-free and overall survival of advanced CRC has improved, but the insensitivity and resistance of chemotherapy drugs is still an important reason for why survival cannot be significantly improved (20). Therefore, the discovery of new targeted treatment sites and the application of new immunotherapeutic methods may achieve the goals of reversing transformational therapy resistance, sensitizing chemotherapeutic drugs, and reducing the dose of chemotherapeutic drugs to reduce the side-effects of chemotherapy (21).

Autophagy is a stress response that is necessary for cell survival (22). Previously published studies have suggested that, during normal conditions, autophagy is kept at a basal level to provide a cytoprotective function, while under negative conditions, such as oxidative stress or hypoxia, uncontrolled autophagy can lead to autophagic death (23). Similarly, autophagy plays context-dependent dual roles in the pathological process of CRC. It has been reported that the expression of LC3 and Beclin-1 is upregulated in CRC, and the enforced expression of LC3 or Beclin-1 is associated with poor prognosis (24-27). In contrast, it has also been reported that Beclin-1 is upregulated by $\gamma$-interferon to 
induce apoptosis in SW480 and HCT116 cells (28).

In the present study, we further confirmed that Ascl2 is upregulated, and is associated with poor prognosis, in CRC. We found that downregulated Ascl2 alleviates proliferation and migration, and promotes apoptosis, in HT-29 cells. To investigate the underlying molecular mechanism of cell death, we examined autophagy proteins, such as LC3 and p62. We found that the suppression of Ascl2 expression leads to excessive autophagy. Cell death, which is induced by downregulating Ascl2, could be counteracted by treatment with autophagy inhibitors, such as 3-MA and CQ. In this context, autophagy could induce cell death in CRC. Finally, we verified the role of Ascl2 in vivo. The findings of the present study indicate that that Ascl2 has tumor-promotion activity by suppressing autophagy to inhibit cell death.

Although the results of the study had been validated in animal experiments and molecular level, it is limited to preliminary exploration. How Ascl2 promotes colorectal cancer tumor progression, as well as its signaling pathway and mechanism is still unclear. Further research is needed.

\section{Acknowledgments}

Funding: This study was supported by the Natural Science Foundation of Minhang, Shanghai (No. 2018MHZ025), Natural Science Foundation of Minhang, Shanghai (No. 2018MHZ037) and the Great Discipline Construction Project from The Fifth People's Hospital of Shanghai (No. 2020WYZDZK02). Leading Talent Foundation of Minhang, Shanghai (No. 10-1).

\section{Footnote}

Reporting Checklist: The authors have completed the ARRIVE reporting checklist. Available at http://dx.doi. org/10.21037/jgo-21-183

Data Sharing Statement: Available at http://dx.doi. org/10.21037/jgo-21-183

Conflicts of Interest: All authors have completed the ICMJE uniform disclosure form (available at http://dx.doi. org/10.21037/jgo-21-183). The authors have no conflicts of interest to declare.

Ethical Statement: The authors are accountable for all aspects of the work in ensuring that questions related to the accuracy or integrity of any part of the work are appropriately investigated and resolved. All procedures performed in this study involving human participants were in accordance with the Declaration of Helsinki (as revised in 2013). The study was approved by Institutional Ethics Committee of The Fifth People's Hospital of Shanghai, Fudan University (No.: 2017-097) and informed consent was taken from all the patients. Animal experiments were performed under a project license (No.: 20171254A184) granted by Department of Laboratory Animal Science, Fudan University, in compliance with Fudan University guidelines for the care and use of animals.

Open Access Statement: This is an Open Access article distributed in accordance with the Creative Commons Attribution-NonCommercial-NoDerivs 4.0 International License (CC BY-NC-ND 4.0), which permits the noncommercial replication and distribution of the article with the strict proviso that no changes or edits are made and the original work is properly cited (including links to both the formal publication through the relevant DOI and the license). See: https://creativecommons.org/licenses/by-nc-nd/4.0/.

\section{References}

1. Sung H, Ferlay J, Siegel RL, et al. Global cancer statistics 2020: GLOBOCAN estimates of incidence and mortality worldwide for 36 cancers in 185 countries. CA Cancer J Clin 2021. [Epub ahead of print]. doi: 10.3322/caac.21660.

2. Thanikachalam K, Khan G. Colorectal Cancer and Nutrition. Nutrients 2019;11:33-44.

3. Rosenbluh J, Nijhawan D, Cox A, et al. b-Catenin-Driven Cancers Require a YAP1 Transcriptional Complex for Survival and Tumorigenesis. Cell 2012;151:1457-73. Erratum in: Cell 2013;153:267-70.

4. Wang C, Wang M, Arrington J, et al. Ascl2 inhibits myogenesis by antagonizing the transcriptional activity of myogenic regulatory factors. Development 2017;144:235-47.

5. van der Flier LG, van Gijn ME, Hatzis P, et al. Transcription Factor Achaete Scute-Like 2 Controls Intestinal Stem Cell Fate. Cell 2009,136:903-12.

6. Tanaka T, Kojima K, Yokota K, et al. Comprehensive Genetic Search to Clarify the Molecular Mechanism of Drug Resistance Identifies ASCL2-LEF1/TSPAN8 Axis in Colorectal Cancer. Ann Surg Oncol 2019;26:1401-11.

7. Shang Y, Chen H, Ye J, et al. HIF-1 $\alpha /$ Ascl2/miR-200b regulatory feedback circuit modulated the epithelialmesenchymal transition (EMT) in colorectal cancer cells. 
Exp Cell Res 2017;360:243-56.

8. Hale AN, Ledbetter DJ, Gawriluk TR, et al. Autophagy: regulation and role in development. Autophagy 2013,9:951-72.

9. Thorburn A. Autophagy and Its Effects: Making Sense of Double-Edged Swords. PLoS Biol 2014;12:e1001967.

10. Singh SS, Vats S, Chia YQ, et al. Dual role of autophagy in hallmarks of cancer. Oncogene 2018;37:1142-58.

11. Lévy J, Cacheux W, Bara MA, et al. Intestinal inhibition of Atg7 prevents tumour initiation through a microbiomeinfluenced immune response and suppresses tumour growth. Nat Cell Biol 2015,17:1062-73.

12. Sato K, Tsuchihara K, Fujii Set al. Autophagy Is Activated in Colorectal Cancer Cells and Contributes to the Tolerance to Nutrient Deprivation. Cancer Res 2007,67:9677-84.

13. Ryter SW, Cloonan SM, Choi AMK. Autophagy: A critical regulator of cellular metabolism and homeostasis. Mol Cells 2013,36:7-16.

14. Jubb AM, Chalasani S, Frantz GD, et al. Achaete-scute like 2 (ascl2) is a target of Wnt signalling and is upregulated in intestinal neoplasia. Oncogene 2006,25:3445-57.

15. Shen T, Cai L, Liu Y, et al. Ube2v1-mediated ubiquitination and degradation of Sirt1 promotes metastasis of colorectal cancer by epigenetically suppressing autophagy. J Hematol Oncol 2018,11:95.

16. Lucas C, Salesse L, Hoang M, et al. Autophagy of Intestinal Epithelial Cells Inhibits Colorectal Carcinogenesis Induced by Colibactin-Producing Escherichia coli in Apc Mice. Gastroenterology 2020,158:1373-88.

17. Ou J, Peng Y, Yang W, et al. ABHD5 blunts the sensitivity of colorectal cancer to fluorouracil via promoting autophagic uracil yield. Nat Commun 2019,10:1078.

18. Nozawa H, Sonoda H, Ishii H, et al. Postoperative chemotherapy is associated with prognosis of stage IV colorectal cancer treated with preoperative chemotherapy/ chemoradiotherapy and curative resection. Int J Colorectal Dis 2020;35:177-80.

19. Zhang C, Tan Y, Xu H. Does adjuvant chemotherapy improve the prognosis of patients after resection of pulmonary metastasis from colorectal cancer? A systematic review and meta-analysis. Int J Colorectal Dis 2019;34:1661-71.

20. Nozawa H, Takiyama H, Hasegawa K, et al. Adjuvant chemotherapy improves prognosis of resectable stage
IV colorectal cancer: a comparative study using inverse probability of treatment weighting. Ther Adv Med Oncol 2019;11:1758835919838960.

21. François S, Usunier B, Forgue-Lafitte ME, et al. Mesenchymal stem cell administration attenuates colon cancer progression by modulating the immune component within the colorectal tumor microenvironment. Stem Cells Transl Med 2019;8:285-300.

22. Zhou H, Yuan M, Yu Q, et al. Autophagy regulation and its role in gastric cancer and colorectal cancer. Cancer Biomark 2016;17:1-10.

23. Sun K, Deng W, Zhang S, et al. Paradoxical roles of autophagy in different stages of tumorigenesis: protector for normal or cancer cells. Cell Biosci 2013,3:35.

24. Guo GF, Jiang WQ, Zhang B, et al. Autophagy-related proteins Beclin-1 and LC3 predict cetuximab efficacy in advanced colorectal cancer. World J Gastroenterol 2011;17:4779-86.

25. Giatromanolaki A, Koukourakis MI, Harris AL, et al. Prognostic relevance of light chain 3 (LC3A) autophagy patterns in colorectal adenocarcinomas. J Clin Pathol 2010;63:867-72.

26. Guo GF, Wang YX, Zhang YJ, et al. Predictive and prognostic implications of 4E-BP1, Beclin-1, and LC3for cetuximab treatment combined with chemotherapy in advanced colorectal cancer with wild-type KRAS: Analysis from real-world data. World J Gastroenterol 2019;25:1840-53.

27. Schmitz KJ, Ademi C, Bertram S, et al. Prognostic relevance of autophagy-related markers LC3, p62/ sequestosome 1, Beclin-1 and ULK1 in colorectal cancer patients with respect to KRAS mutational status. World J Surg Oncol 2016;14:189.

28. Wang QS, Shen SQ, Sun HW, et al. Interferon-gamma induces autophagy-associated apoptosis through induction of cPLA2-dependent mitochondrial ROS generation in colorectal cancer cells. Biochem Biophys Res Commun 2018;498:1058-65.

(English Language Editor: R. Scott)

Cite this article as: Wang H, Ye T, Cai Y, Chen W, Xie H, Ke C. Downregulation of Ascl2 promotes cell apoptosis by enhancing autophagy in colorectal cancer cells. J Gastrointest Oncol 2021;12(2):630-638. doi: 10.21037/jgo-21-183 